\title{
Staphylococcus aureus adquiridos en la comunidad: caracterización clínica, fenotípica y genotípica de aislados en niños paraguayos
}

\author{
Rosa Guillén, Letizia Carpinelli, Fátima Rodríguez, Héctor Castro, Beatriz Quiñónez, \\ Ana Campuzano, María Macchi, Juana Ortellado, Patricia Almada, Lorena Grau, Mónica Rodríguez, \\ Gladys Velázquez, Carmen Espínola, Gloria Samudio, Gloria Gómez y Wilma Basualdo
}

Community-acquired Staphylococcus aureus isolated from Paraguayan children: clinical, phenotypic and genotypic characterization

Introduction: The prevalence of Staphylococcus aureus in the community has increased, being the pediatric population the most affected. This fact highlights the need for epidemiological surveillance. Aim: To characterize clinical, phenotypic and genotypic isolates of $S$. aureus children's samples with community-acquired infections, collected in hospitals of Asuncion and the Central Department, between November 2009 and December 2010. Materials and Methods: Descriptive and transverse analysis with analytical component. Clinical data collected by medical records, antibiotic susceptibility according to CLSI criteria and detection of $m e c A$ (encoding methicillin resistance) and $l u k-P V$ genes (encoding Panton Valentine leucocidin) by PCR using specific oligonucleotides. Results: 123 isolates of $S$. aureus, $76 \%$ came from skin and soft tissue infections and $20 \%$ from sepsis. $18.7 \%$ $(\mathrm{n}=23)$ were resistant to methicillin (MRSA). The presence of the mecA gene, a variant there and the PVL was detected in 12.2 and 48 isolates respectively. $43 \%$ of MRSA $(\mathrm{n}=10)$ was carrying $l u k-P V$. The clinical and demographic differences between patients infected with MRSA or MSSA were not statistically significant. Discussion: This study constitutes the first phenotypic and genotypic characterization of $S$. aureus associated with pediatric patients in Paraguay.

Key words: Community-acquired Staphylococcus aureus, mecA, PVL, pediatric population.

Palabras clave: Staphylococcus aureus adquiridos de la comunidad, mecA, PVL, población pediátrica.

\section{Introducción}

$\int \mathrm{g}$ taphylococcus aureus es un microorganismo patógeno para el hombre, capaz de causar una multitud de infecciones con un amplio rango de gravedad, desde infecciones localizadas de piel e intoxicaciones alimentarias, hasta infecciones invasoras y potencialmente mortales como neumonía necrosante, osteomielitis y sepsis $^{1,2}$. Su diversidad patogénica refleja su habilidad para colonizar exitosamente, adaptarse y sobrevivir en diferentes tejidos celulares durante la infección, lo que puede deberse a su capacidad de portar genes que le confieren resistencia a antimicrobianos, otros que codifican factores de virulencia, y su plasticidad genética; todo esto podría contribuir a una progresión más rápida y complicada de la enfermedad bajo determinados factores ambientales, ya que permiten una mejor adaptación al hospedero ${ }^{3,4}$.

Staphylococcus aureus ha desarrollado resistencia a prácticamente todas las clases de antimicrobianos, principalmente a los $\beta$-lactámicos. El mecanismo más frecuente de resistencia a los $\beta$-lactámicos radica en la penicilinasa, enzima que hidroliza a la penicilina y a otros compuestos sensibles. Los primeros $\beta$-lactámicos estables frente a la acción de penicilinasa como las cefalosporinas y meticilina estuvieron disponibles a finales de 1950 sólo unos años después se reportó la aparición de las primeras cepas resistentes a meticilina ${ }^{5,6}$. Desde su aparición en Inglaterra en 1961, la incidencia de $S$. aureus resistente a meticilina (SARM) ha ido en constante aumento y es así como se ha constituido en uno de los patógenos prevalentes en infecciones nosocomiales. Sin embargo, la epidemiología de SARM fue modificándose con el tiempo y actualmente se observa un incremento de infecciones adquiridas en la comunidad en pacientes sin factor de riesgo conocido $0^{5,7-9}$. Los aislados de SARM adquiridos en la comunidad (SARM-AC) poseen diversas características que los distinguen de las cepas nosocomiales; así por ejemplo, presentan resistencia a menos antimicrobianos que las cepas de origen hospitalario ${ }^{10-12}$. Las infecciones asociadas más frecuentemente a SARM-AC son las relacionadas con piel y tejidos blandos (85-90\% de los casos), y en menos de $10 \%$ causan infecciones graves (ej. estafilococcemia,
Instituto de Investigaciones en Ciencias de la Salud, UNA. Departamento de Análisis Clínicos y Microbiología. Departamento de Biología Molecular y Biotecnología (RG, LC, FR).

Hospital General Pediátrico Niños de Acosta Ñú. Departamento de Epidemiología y Enfermedades Infecciosas. Laboratorio de Microbiología (HC, BQ, WB).

Hospital de Clínicas, FCM, UNA Cátedra de Pediatría y Laboratorio de Microbiología (AC, MM, JO, PA) Hospital Central, Dpto. de Epidemiología y SIS. Instituto de Previsión Social. Servicio de Pediatría y Laboratorio de Microbiología (LG, MR, GV, CE). Hospital Nacional de Itauguá. Servicio de Pediatría y Servicio de Microbiología (GS, GG).

Los autores declaran no tener conflictos de interés. El trabajo fue financiado con el premio LabCiencia otorgado en el VI Congreso Paraguayo de Química, llevado a cabo en junio de 2008 en Asunción, Paraguay.

El componente de análisis molecular ha sido realizado con reactivos del Departamento de Biología Molecular y Biotecnología del IICSUNA y con financiación obtenida del premio LAB Ciencia del VI Congreso Paraguayo de Química 2008.

Recibido: 24 de mayo de 2016 Aceptado: 12 de agosto de 2016

Correspondencia a: Wilma Basualdo wilmabasualdo@gmail.com 
neumonía necrosante y osteomielitis) que frecuentemente se asocian a alta morbilidad y mortalidad ${ }^{9,13,14}$.

La resistencia a meticilina en $S$. aureus ha sido asociada a la presencia de un elemento genético móvil (SCCmeccassette cromosómico estafilocóccico que confiere resistencia a meticilina), dentro del cual se inserta el gen $m e c A$. Éste codifica una proteína de unión a penicilina, PBP2a, con actividad transpeptidasa responsable de la resistencia intrínseca a todos los $\beta$-lactámicos ${ }^{2}$.

Además, se ha descrito que SARM-AC presenta diversos factores de virulencia, principalmente la leucocidina de Panton-Valentine (PVL-Panton Valentine leukocidin), cuyo gen codificante se ha visto asociado al SCCmec tipo IV y V, relacionados con los casos de SARM$\mathrm{AC}^{2,15,16}$. Por lo general, en estos aislados, la resistencia a oxacilina no está acompañada por la resistencia a otros antimicrobianos, excepto algunas cepas donde se observa resistencia a eritromicina, clindamicina, cloranfenicol y gentamicina $^{15,17}$.

En la primera década del siglo veintiuno, diversas publicaciones comunicaron aislamiento de SARM-AC en población pediátrica alrededor del mundo ${ }^{2,8,18}$. En E.U.A., la prevalencia de SARM-AC varía dependiendo de la localización geográfica entre 26 y $62 \%{ }^{19}$. Los primeros estudios en Latinoamérica fueron reportados por Uruguay en el año $2003^{20}$. En el año 2005, H. Paganini y cols., de la Argentina, comunicaron una prevalencia de SARM-AC de $42 \%$. Esta cifra aumentó considerablemente durante los años siguientes, siendo en el 2009 , de $61 \%{ }^{15}$. Igualmente, en un estudio realizado por Reyes y cols., en el año 2009, de cepas de SARM en países de Latinoamérica, se reportó una prevalencia de SARM-AC que oscilaba entre 26 y $62 \%{ }^{21}$.

En nuestro país, estudios provenientes de la Red de Vigilancia a Antimicrobianos del Laboratorio Central de Salud Pública (LCSP), reportaron una prevalencia de SARM-AC de $25 \%$ en el año 2008 y $32 \%$ para el año 2013 en la población general. Igualmente, los hospitales pediátricos de referencia del país, reportan una prevalencia de SARM-AC que oscila entre 10 y $27 \%$, de acuerdo al centro asistencial, observándose un incremento en la prevalencia de SARM-AC, en los últimos años ${ }^{22-25}$.

El manejo de las infecciones causadas por SARM-AC, en población pediátrica, representa actualmente un desafío en todas las instituciones sanitarias. El conocimiento de la prevalencia de SARM-AC y los factores de virulencia, serán de utilidad para el manejo de las infecciones en la población pediátrica.

El presente estudio se realizó con el objetivo principal de caracterizar desde el punto de vista clínico, fenotípico y genotípico aislados de $S$. aureus obtenidos de muestras biológicas de niños con infecciones adquiridas en la comunidad, que concurrieron a hospitales de referencia de Asunción y el Departamento Central.

\section{Materiales y Métodos}

\section{Diseño del estudio y población}

Observacional, descriptivo con componente analítico, de corte trasversal, llevado a cabo entre noviembre de 2009 y diciembre de 2010, con la participación de los siguientes centros de referencia: Hospital General Pediátrico Niños de Acosta Ñú (HGP), Hospital de Clínicas (HCL), Hospital Central del Instituto de Previsión Social (IPS) y Hospital Nacional de Itauguá (HNI). La población estudiada incluyó aislados de $S$. aureus obtenidos de fluidos corporales, secreción de piel y tejidos blandos de pacientes bajo 18 años de edad, con cuadro clínico compatible con infecciones por $S$. aureus adquiridas en la comunidad. Fueron excluidos del trabajo aquellos aislados obtenidos a partir de secreción traqueal, punta de catéter e hisopados, así como aquellos que provenían de pacientes con18 años de edad o más.

\section{Definición de infección por S. aureus adquirida en la comunidad}

Se consideró como infección adquirida en la comunidad, aquella que fue diagnosticada en las primeras $72 \mathrm{~h}$ de la internación, con documentación microbiológica y además que el paciente no presentara factores de riesgo como: hemodiálisis, cirugía, presencia de una sonda permanente o de un dispositivo percutáneo en el momento del cultivo.

\section{Datos clínicos}

Estos fueron registrados en una ficha especialmente diseñada para el efecto por los centros participantes. Las variables estudiadas incluyeron: edad, sexo, procedencia, diagnóstico de egreso, manejo del paciente y pronóstico. Así mismo, se recolectaron datos asociados a factores que favorecen la infección por $S$. aureus como: número de convivientes, tratamiento previo con antimicrobianos u hospitalización anterior a la infección, exposición a procedimientos médicos invasores y presencia de comorbilidad.

\section{Aislados de S. aureus}

La totalidad de los aislados incluidos en este estudio fueron obtenidos a partir de muestras biológicas remitidas para su cultivo a los servicios de microbiología de los hospitales participantes del estudio: HGP, HCL, IPS y HNI. Las muestras biológicas fueron analizadas siguiendo las recomendaciones de los manuales de procedimiento de cada servicio de microbiología. La prueba de susceptibilidad a antimicrobianos se realizó por el método de difusión de disco, así como la determinación de la concentración inhibitoria mínima (CIM) mediante el empleo de métodos automatizados (Vitek, BioMérieux, Francia). Para la detección de la resistencia inducible a 
clindamicina, se realizó la prueba de D-test empleando la difusión de doble disco. En todos los casos se siguieron las recomendaciones del Clinical and Laboratory Standard Institute (CLSI). Los aislados duplicados del mismo paciente fueron excluidos. Los aislados que cumplieron los criterios de inclusión fueron mantenidos congelados en un medio criopreservante hasta la remisión al IICS.

Fueron excluidos del estudio aquellos aislados de $S$. aureus provenientes de puntas de catéter, secreción traqueal, así como aquellos que no presentaban datos clínicos completos o con falta de identificación y conservación apropiadas. La detección de la proteína PBP2a se realizó en algunos aislados de $S$. aureus empleando la prueba de latex PBP2' Test Kit de Oxoid, Japón.

\section{Detección de genes mecA y luk-PV}

La extracción de ADN se realizó a partir de aislados criopreservados remitidos por los servicios de microbiología, realizando una suspensión bacteriana correspondiente a 1 en la escala de Mac Farland con $200 \mathrm{~mL}$ de agua miliQ estéril (milipore, E.U.A.) por el método de ebullición descrito por Zhang y cols. ${ }^{26}$. Para las reacciones de amplificación se emplearon como molde $2,5 \mathrm{~mL}$ del sobrenadante obtenidos en la extracción de ADN. La amplificación del gen $m e c A$ se realizó empleando oligonucleótidos y condiciones de ciclado descritos por Murakami y cols. ${ }^{27}$, obteniéndose un producto de $533 \mathrm{pb}$. La amplificación del gen codificante de la PVL se realizó empleando oligonucleótidos y condiciones de ciclado descritos por Lina y cols. ${ }^{28}$ y se obtuvo un producto de 433 pb. En aquellos aislados en los que se observó discordancia entre el fenotipo de resistencia a meticilina y la presencia del gen $m e c A$, se realizó una reacción de polimerasa en cadena (RPC) para detectar una variante de dicho gen descrita por García-Álvarez y cols., en el 2011, cuya secuencia presenta diferencias que impiden el anillamiento de los oligonucleótidos descritos por varios autores ${ }^{29}$.

En cada RPC se incluyó controles negativos y controles positivos (cepa ATCC 43300 portadora del gen mecA y un aislado gentilmente cedido por Gabriel Gutkind de la Facultad de Farmacia de la UBA, Argentina, portador de $m e c A$ y $l u k-P V)$. Los productos de RPC se visualizaron en geles de agarosa al $2 \%$ con tinción de bromuro de etidio. Se empleó como marcador de peso molecular 100 bp DNA ladder (Bioline, E:U.A.). El tamaño de los productos amplificados fue determinado mediante el uso del software Kodak Digital Science. Algunos productos de cada reacción fueron seleccionados al azar y enviados para su secuenciación a la empresa Macrogen Inc. (Corea). Las secuencias obtenidas fueron alineadas con la base de datos del NCBI para corroborar su identidad, obteniéndose porcentajes de concordancia en los alineamientos mayores a $95 \%$ en las muestras analizadas.

\section{Aspectos éticos}

El protocolo de estudio fue sometido y aprobado por el Comité Científico y Ético del Instituto de Investigaciones en Ciencias de la Salud, UNA, y por las autoridades de los hospitales participantes. Los datos personales de los pacientes fueron mantenidos en estricta confidencialidad, siendo manejados con códigos. Las muestras biológicas analizadas fueron parte del esquema de rutina de diagnóstico para un cuadro infeccioso en los pacientes y no se tomaron muestras adicionales para el estudio. Los médicos tratantes recibieron los resultados del análisis microbiológico necesario para orientar el tratamiento apropiado de la infección. Los resultados moleculares no inciden en el tratamiento implementado y su beneficio es principalmente en el área epidemiológica para identificar la frecuencia de aislados de $S$. aureus portadores del gen $m e c A$ y luk- $P V$ que circulan en los hospitales de referencia participantes.

\section{Análisis estadísticos}

Los datos fueron introducidos y almacenados en una base de datos, utilizando una planilla electrónica (Microsoft Excel). El análisis descriptivo se realizó empleando los software Epi Info 3.5.0. Para variables categóricas el análisis se realizó usando $\chi^{2}$ y el test exacto de Fischer. El nivel de significancia estadística se consideró con valores de $\mathrm{p}<0,05$.

\section{Resultados}

Se estudiaron 123 aislados de $S$. aureus remitidos respectivamente por el HGP (n: 48, 39\%), el HCL (n: 43, 35\%), IPS (n: $26,21 \%$ ) y HNI (n: 6, 5\%). La mayor frecuencia de aislamiento de $S$. aureus se dio en el período de diciembre de 2009 a marzo de 2010, correspondiendo a $62 \%$ del total analizado (n: 76). Los aislados provinieron de 56 niñas $(45,5 \%)$ y 67 niños $(54,5 \%)$ con una mediana de edad de 60 meses (rango 1 día a 18 años). Los grupos etarios que presentaron los mayores porcentajes de infección por $S$. aureus fueron: de 1 a 4 años y de 5 a 14 años con 36,6 y 38,2\%, respectivamente (Figura 1). El $66,6 \%$ de los pacientes residía en el Departamento Central, seguido de Asunción (9,8\%). Los departamentos de San Pedro, Cordillera, Caaguazú y Paraguarí aportaron en conjunto 17 y $6,5 \%$ de las muestras. Las muestras restantes provenía de niños que residían en alguno de los siguientes departamentos: Caazapá, Canindeyú, Itapúa, Misiones, Pdte Hayes, Alto Paraná y Guairá.

Dentro de factores predisponentes a infecciones por $S$. aureus observados se encuentran: el empleo previo de antimicrobianos en $16 \%$ de los niños (n: 17), la presencia de co-morbilidad en $8,9 \%$ (n: 11) y la convivencia con un trabajador de la salud como miembro de la familia en 
2,4\% (n: 3). Presentaron co-morbilidad cinco niños con enfermedades pulmonares crónicas, dos niños con patología cutánea crónica, dos niños con leucemia linfoblástica aguda (LLA) y un niño con lupus eritematoso diseminado (LES). Así mismo, el número de convivientes presentó una mediana de cinco y valores extremos de entre dos y

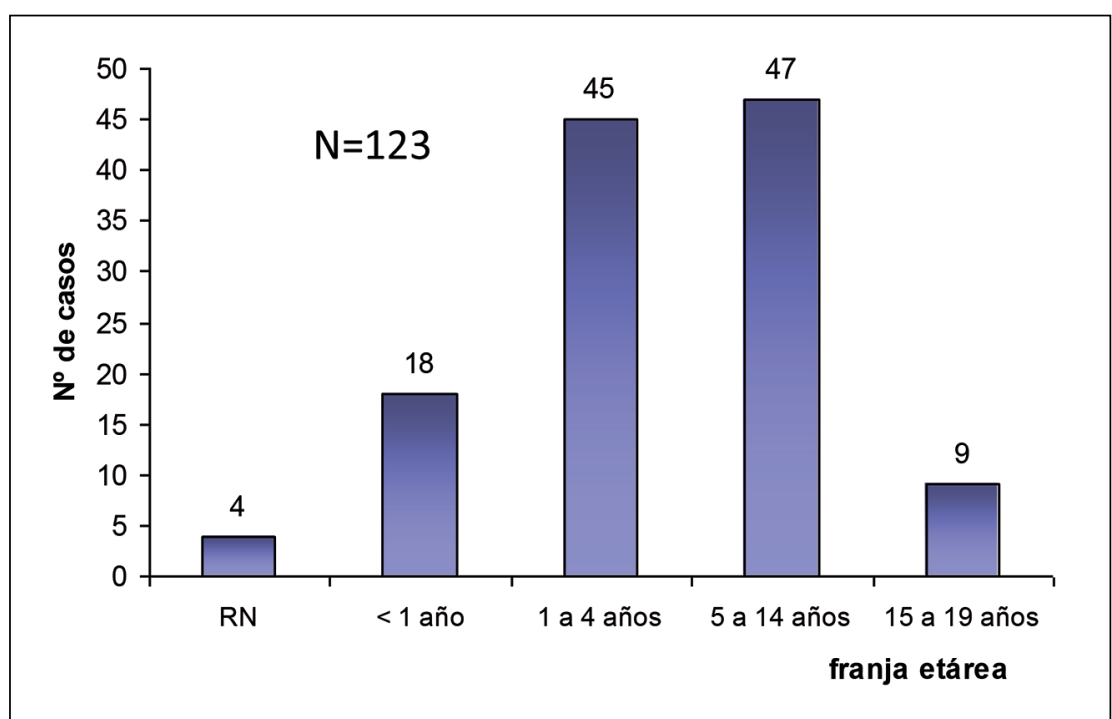

Figura 1. Distribución de casos de infección con S. aureus según franja etárea.

Tabla 1. Características clínicas y demográficas de pacientes con infección por S. aureus

\begin{tabular}{|ll|}
\hline Características & $\begin{array}{l}\text { Número (\%) } \\
\mathbf{n}=123\end{array}$ \\
\hline Edad en meses* & $60(1$ día- 18 años) \\
\hline Sexo masculino & $67(54,5)$ \\
\hline$N^{\circ}$ de convivientes* & $5(2-15)$ \\
\hline Co-morbilidad & $11(8,9)$ \\
\hline Trabajador de salud en la familia & $3(2,4)$ \\
\hline Tratamiento previo con antimicrobiano & $17(16)$ \\
\hline Internación previa & $21(17)$ \\
\hline Cuadro clínico & \\
Infección de piel y tejidos blandos & $94(76)$ \\
Sepsis con focos múltiples & $18(15)$ \\
Osteomielitis y artritis & $3(2,4)$ \\
Neumonía & $3(2,4)$ \\
Otitis supurada & $2(2)$ \\
Sepsis & $2(2)$ \\
Absceso hepático & $1(1)$ \\
\hline *Mediana (rango). & \\
\hline
\end{tabular}

15 personas viviendo con el niño infectado; no podemos, sin embargo, definir las condiciones de hacinamiento ya que no contamos con datos de la distribución en espacio de las viviendas de los pacientes (Tabla 1).

El 76\% (n: 94) de los niños presentó cuadros clínicos compatibles con infecciones de piel y tejidos blandos, mientras que se observó una frecuencia mucho menor de otros cuadros clínicos como sepsis, osteomielitis, neumonía, otitis y absceso hepático (Tabla 1). El 19,5\% $(24 / 123)$ presentó bacteriemia. Se empleó como tratamiento drenaje acompañado de antibioterapia en $70,7 \%$ de los niños (n: 87). El 88,6\% (n: 109) mejoró en respuesta al tratamiento implementado. Cuatro pacientes fallecieron $(3,3 \%)$, presentando todos cuadro clínico de sepsis con foco múltiple; dos presentaron como co-morbilidad asociada LES y LLA.

Los aislados de $S$. aureus fueron obtenidos a partir de 96 secreciones purulentas de piel y tejidos blandos $(78,0 \%), 23$ muestras de sangre $(19,7 \%)$, dos de secreciones de oído, una de hueso y uno de líquido articular.

No se detectaron aislados resistentes a tigeciclina y teicoplanina, si bien estos antimicrobianos fueron ensayados en $77(62,6 \%)$ y $12(9,8 \%)$ de los aislados, respectivamente. Veintitrés cepas de $S$. aureus fueron resistentes a meticilina $(18,7 \%)$.

Comparando los perfiles de resistencia a antimicrobianos de los aislados SARM respecto a las cepas de $S$. aureus sensibles a meticilina (SASM) (Tabla 2), se observó que los aislados SARM presentaron niveles mayores de resistencia a ciprofloxacina, clindamicina, eritromicina y gentamicina respecto a las cepas de SASM, resultando una diferencia estadísticamente significativa la que se observó con gentamicina (p: 0,021, test exacto de Fischer). Así mismo se registraron niveles de resistencia menores de 3,5\% para tetraciclina, cotrimoxazol y rifampicina en SASM, si bien las diferencias con los aislados de SARM para estos antimicrobianos no fueron estadísticamente significativas. El antibiotipo más frecuente en SASM es el de resistencia a penicilina (59\% de los casos), mientras que en SARM, $69,6 \%$ presentó resistencia a dos antimicrobianos, siendo la combinación más frecuente la resistencia a oxacilina y penicilina (n: $14 ; 60,8 \%$ ) seguida de las combinaciones de resistencia a oxacilina con gentamicina y oxacilina con eritromicina ( $4 \%$ en ambos casos) (Tabla 3). La resistencia a clindamicina fue detectada en 5,7\% (n: 7) de las cepas, dos de estos aislados eran SARM.

El análisis univariado comparando pacientes con SARM y SASM respecto a variables como edad, sexo, tipo de infección, co-morbilidad y presencia de factores predisponentes, no fue estadísticamente diferente ( $p>0,05, \chi^{2}$ o test exacto de Fischer).

La RPC para la detección de los genes mec $A$ y luk- $P V$ generó los productos de tamaño esperado (Figura 2). La totalidad de los aislados de $S$. aureus fue sometida a la 


\begin{tabular}{|c|c|c|c|c|c|c|c|c|c|}
\hline \multirow[b]{2}{*}{ Antibiótico } & \multicolumn{3}{|c|}{ SARM $^{a}$} & \multicolumn{2}{|c|}{ SASM ${ }^{b}$} & \multicolumn{3}{|c|}{ Total $^{c}$} & \multirow[b]{2}{*}{$\mathbf{p}^{\mathrm{e}}$} \\
\hline & $\mathbf{N}$ & & $\mathbf{R}^{\mathbf{d}}$ & $\mathrm{n}$ & $\mathbf{R}^{\mathrm{d}}$ & $\mathrm{N}$ & & $R^{d}$ & \\
\hline Penicilina & 18 & 18 & (100) & 75 & 72 (96) & 93 & 90 & $(96,6)$ & 0,502 \\
\hline Cefoxitina & 19 & 19 & (100) & 64 & $0 \quad(0)$ & 82 & 19 & $(15,9)$ & 0,000001 \\
\hline Ciprofloxacina & 23 & 1 & $(4,3)$ & 98 & $1 \quad(1)$ & 121 & 2 & $(1,65)$ & 0,345 \\
\hline Clindamicina & 20 & 20 & $(10)$ & 81 & $5 \quad(6,2)$ & 101 & 7 & $(6,9)$ & 0,420 \\
\hline Eritromicina & 21 & 4 & (19) & 85 & $7 \quad(8,2)$ & 116 & 8 & $(6,9)$ & 0,145 \\
\hline Gentamicina & 20 & 3 & (15) & 85 & $1 \quad(1,2)$ & 105 & 4 & $(3,8)$ & 0,021 \\
\hline Tetraciclina & 21 & 0 & $(0)$ & 73 & $(2,7)$ & 94 & 2 & $(2,1)$ & 0,601 \\
\hline Cotrimoxazol & 20 & 0 & (0) & 80 & $(3,8)$ & 100 & 3 & $(3,0)$ & 0,508 \\
\hline Rifampicina & 20 & 0 & (0) & 81 & $(3,8)$ & 101 & 3 & $(2,98)$ & 0,511 \\
\hline
\end{tabular}

detección de ambos genes. Se detectó la presencia de mec $A$ y luk- $P V$ en $12(9,76 \%)$ y $48(39,0 \%)$ de los aislados, respectivamente. La presencia de ambos genes de forma simultánea se dio en 10 aislados $(8,1 \%$ del total de aislados y $43,5 \%$ del total de SARM). La totalidad de las cepas portadoras de mecA y luk- $P V$ fue causante de infecciones de piel y tejidos blandos (n: 12), mientras que de los dos aislados que sólo presentaron mec $A$ provenían de muestras colectadas de un caso de sepsis y una infección de piel y tejidos blandos.

No se detectó el gen $m e c A$ en aislados previamente tipificados como sensibles a oxacilina por métodos fenotípicos. Sin embargo, estuvo presente en 12 del total de 23 aislados caracterizados como SARM por métodos fenotípicos.

La no concordancia de $100 \%$ entre los resultados de la RPC y la tipificación fenotípica nos llevó a plantear la posibilidad de la presencia de una variante en la secuencia del gen que impida la unión de los oligonucleótidos descritos por Murakami, o bien la presencia de otro mecanismo responsable del fenotipo de resistencia a meticilina. Por lo anteriormente expuesto, se repitieron los antibiogramas de los 11 aislados en el laboratorio de microbiología del IICS y observamos que la totalidad mostraba un fenómeno de heteroresistencia respecto al disco de oxacilina. Seleccionamos de forma aleatoria uno de estos aislados y realizamos la prueba de latex para detección de la presencia de la proteína PBP2a, obteniendo aglutinación positiva pero menos intensa que la observada en la cepa empleada como control positivo. Los 11 aislados fueron entonces sometidos a amplificación por RPC empleando oligonucleótidos degenerados descritos por García-Álvarez y cols. Dichos oligonucleótidos tienen variaciones en su secuencia que permiten amplificar $m e c A$ y genes homólogos aun cuando la similitud de secuencia no sea perfecta. Dos de los once aislados generaron el producto esperado según
Tabla 3. Frecuencia de antibiotipos observados de aislados de SARM y SASM

\begin{tabular}{lclc}
\hline Patrón de resitencia* & $\begin{array}{c}\text { SASM } \\
\mathbf{n}(\%)\end{array}$ & Patrón de resistencia* & $\begin{array}{c}\text { SARM } \\
\mathbf{n}(\%)\end{array}$ \\
\hline Pen & $59(59)$ & Oxa & $2(8,7)$ \\
Tet & $1(1)$ & Oxa+Pen & $14(60,9)$ \\
Pen+Rif & $3(3)$ & Oxa+Gen & $1(4,3)$ \\
Pen+Eri & $3(3)$ & Oxa+Eri & $1(4,3)$ \\
Pen+Clin & $3(3)$ & Oxa+Pen+Genta & $1(4,3)$ \\
Pen+Cip & $1(1)$ & Oxa+Pen+Eri & $1(4,3)$ \\
Pen+Sxt & $1(1)$ & Oxa+Gen+Eri & $1(4,3)$ \\
Pen+Tet & $1(1)$ & Oxa+Pen+Eri+Cip & $1(4,3)$ \\
Clin+Eri & $2(2)$ & Oxa+Pen+Eri+Gen & $1(4,3)$ \\
Pen+Eri+Sxt & $1(1)$ & & \\
Pen+Eri+Sxt+Clin & $1(1)$ & &
\end{tabular}

*Pen: Penicilina, Oxa: Oxacilina, Gen: Gentamicina, Eri: Eritromicina, Clin: Clindamicina, Sxt: Cotrimoxazol, Tet: Tetraciclina, Cip: Ciprofloxacina, Rif: Rifampicina.

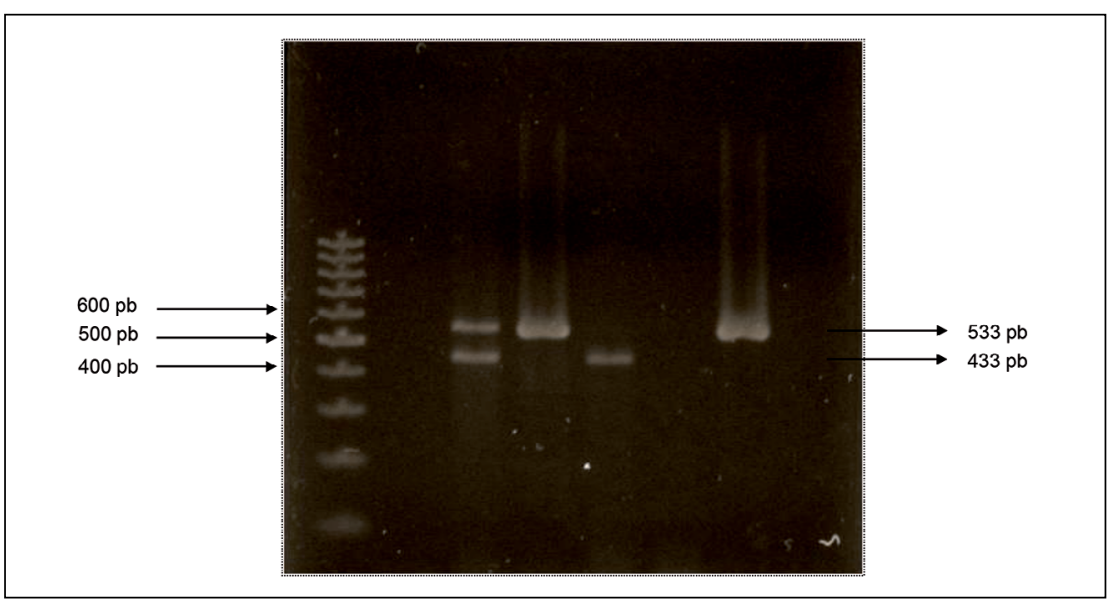

Figura 2. RPC múltiple para la detección de genes mecA y luk-PV. Gel de agarosa al $2 \%$ teñido con bromuro de etidio. Carriles de izquierda a derecha: 1. Marcador de 100pb; 2. Control negativo de la reacción; 3. Control positivo cepa portadora de genes mecA y pvl; 4. Cepa ATCC43300, control positivo para mecA; 5. Aislado SCM55 6. Aislado SIP10; 7. Aislado SCM44; 8. Aislado SGP58. 


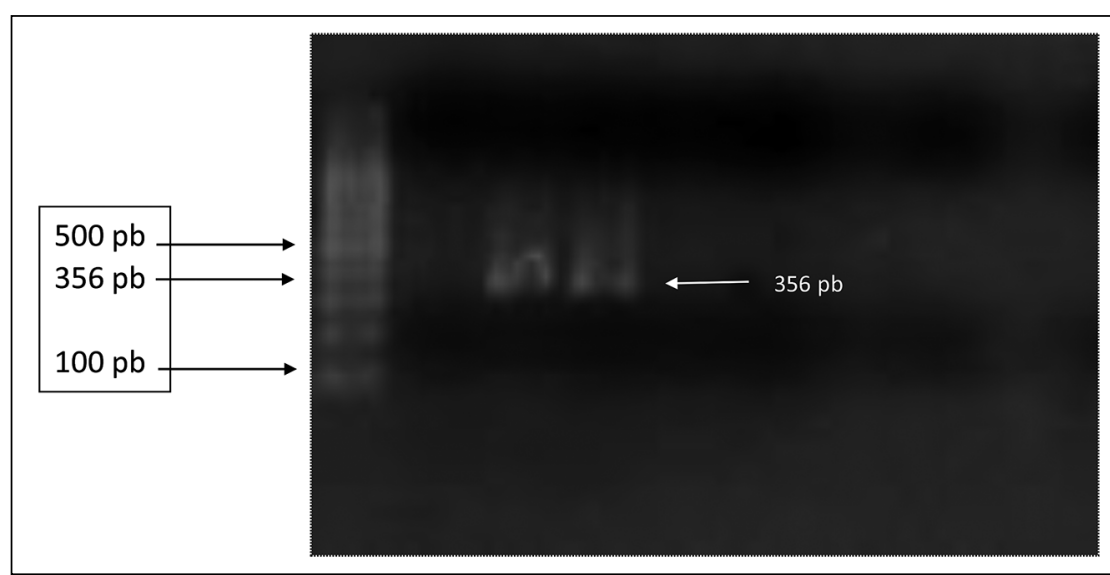

Figura 3. RPC para detección del gen mecC empleando oligonucleótidos degenerados descritos por García-Alvarez. Gel de agarosa al 2\% teñido con bromuro de etidio. Carriles de izquierda a derecha: 1: Marcador de peso molecular de 100 pb; 2: Control negativo; 3 al 9: Aislados con heterorresistencia frente a discos de oxacilina que resultaron negativos para la RPC convencional para mecA descrita por Murakami y cols. Producto esperado para el gen mecC: $356 \mathrm{pb}$.

Tabla 4. Análisis univariado del tipo de infecciones generadas por SASM-PVL(-) versus SASM-PLV(+)

\begin{tabular}{lcccc}
\hline Tipo de infección & $\begin{array}{c}\text { PVL negativo } \\
(\mathbf{n}=\mathbf{6 2})\end{array}$ & $\begin{array}{c}\text { PVL positivo } \\
(\mathbf{n}=\mathbf{3})\end{array}$ & RR (IC) & $\mathbf{p}$ \\
\hline $\begin{array}{l}\text { Infecciones de piel y tejidos blandos } \\
(\mathbf{n}=76)\end{array}$ & $49(79 \%)$ & $27(71 \%)$ & $0,90(0,71-1,14)$ & 0,36 \\
\hline $\begin{array}{l}\text { Infecciones invasoras } \\
\left(\mathbf{n}=22^{*}\right)\end{array}$ & $11(18 \%)$ & $11(29 \%)$ & $1,88(0,9-3,8)$ & 0,08 \\
\hline
\end{tabular}

*No se incluyen 2 cepas de otitis supurada. sistencia constituya un pilar fundamental para el manejo de estos pacientes ${ }^{19-22}$. En nuestro estudio observamos que $18,7 \%$ de las infecciones adquiridas en la comunidad fueron producidas por cepas de SARM. Estos resultados son inferiores a los reportados por estudios realizados en otros centros hospitalarios de nuestro país, donde se comunica una prevalencia que oscila entre 23 y $27 \% \%^{23,24}$.

El mayor porcentaje de aislamiento se observó entre diciembre y marzo, coincidente con el verano, caracterizado por el calor intenso y las picaduras de insectos, con un aumento en la frecuencia de infecciones de piel y tejidos blandos. Las elevadas temperaturas han sido señaladas como un factor ambiental importante en el desarrollo de infecciones por $S$. aureus ${ }^{30}$.

Los datos mostraron que el empleo previo de antimicrobianos y la presencia de alguna co-morbilidad fueron los mayores factores predisponentes a infecciones por $S$. aureus en los niños estudiados. La convivencia con un trabajador de la salud como miembro de la familia se dio apenas en 2,4\% (n: 3). Dentro de la co-morbilidad, cinco niños tenían enfermedades pulmonares crónicas, dos niños padecían de LLA, dos con patología cutánea crónica y un paciente tenía LES. La presencia de alguna co-morbilidad resulta muy importante en la evolución del cuadro clínico; de hecho $50 \%$ de los óbitos registrados se dieron en niños con enfermedades como LES y LLA. El número de convivientes presentó una mediana de cinco y valores extremos dos a 15 personas viviendo con el niño infectado, hallazgo similar al descrito en estudios realizados a nivel internacional y nacional ${ }^{9,23,24}$.

Las infecciones de piel y tejidos blandos representaron más de la tercera parte de los casos estudiados y $19,5 \%$ de los pacientes presentaba bacteriemia, datos que coinciden a los presentados por Sanabria y cols., donde $62 \%(112 / 180)$ de las infecciones eran de piel y tejidos blandos ${ }^{24}$. En Córdoba, Argentina, un estudio realizado en 14 hospitales mostró que SARM-AC estaba asociado con mayor frecuencia a infecciones de piel y tejidos blandos que SARM de adquisición hospitalaria, $\mathrm{p}<0,001^{31}$.

La frecuencia de los aislados SARM (18,7\%, n: 23) hallada en población pediátrica en el presente trabajo es menor a la reportada a por la Red Nacional de Monitoreo de Resistencia a Antimicrobianos (32\%) en la población general, así como también es inferior la encontrada en un estudio realizado en octubre de 2009 en personal de salud del Hospital General Pediátrico Niños de Acosta Ñú $(31,7 \%)^{22,32}$. Esta diferencia observada, obedece al tipo de poblaciones analizadas y resalta la importancia del presente estudio, en el que se caracteriza por vez primera aislados de $S$. aureus adquiridos en la comunidad en población pediátrica.

La totalidad de las cepas de SARM y SASM analizadas en este estudio fueron sensibles a vancomicina, 
tigeciclina y teicoplanina. Las frecuencias de resistencia a tetraciclina, rifampicina y cotrimoxazol permanecen por debajo de $4 \%$ de los aislados, mientras que la resistencia a clindamicina se detectó en $5,7 \%$ de los casos. Estos resultados coinciden con lo reportado en trabajos realizados tanto en Paraguay como en Argentina ${ }^{9,23,24,32,33}$. La resistencia a penicilina es la más frecuente, tanto en SARM como en SASM, mientras que la resistencia a gentamicina es mayor en los aislados de SARM que en los de SASM, hechos coincidentes con otros estudios ${ }^{24,32}$. Diversos autores han reportado que el clon pediátrico circulante en Latinoamérica se caracteriza por causar infecciones en niños y presentar resistencia limitada a $\beta$-lactámicos asociada a resistencia a gentamicina ${ }^{34}$. Para poder confirmar la presencia de este clon entre los estudiados en este trabajo precisamos realizar análisis moleculares como la tipificación del cassette SCCmec, MLST y Spa typing, los que planeamos hacer en un futuro próximo. Los porcentajes de resistencia a tetraciclina son muy inferiores a los reportados en un estudio que analizó el comportamiento de cepas de S. aureus del complejo clonal 80, proveniente de 14 países europeos, seis africanos, dos del Medio Oriente y uno de Asia, en la que describen $70 \%$ de resistencia a tetraciclina en aislados de SARM; los autores sugieren que esta característica ha sido seleccionada a través de plásmidos presentes en linajes ancestrales ${ }^{35}$.

La detección de los genes mecA y luk- $P V$ se dio en 9,8\% (n: 12) y $31 \%$ (n: 38 ) de los aislados, respectivamente. Cerca de la mitad de los aislados SARM presentó también el gen $p v l$. Así como en otros países, en este trabajo la totalidad de los aislados SARM portadores de los genes mec y luk-PV causaron infecciones de piel y tejidos blandos ${ }^{10,14,31}$.

Tanto en Dinamarca como en el Reino Unido, diversos grupos de investigación han reportado en junio del año 2011, nuevos homólogos del gen mecA, que presentan diferencias en la secuencia de ADN y que no son detectables por métodos moleculares descritos con anterioridad, como es el caso del protocolo descrito por Murakami y que fue utilizado en este trabajo ${ }^{29,36}$. Por este motivo analizamos la posibilidad de la presencia de un homólogo del gen mecA en aquellos 11 aislados previamente tipificados como resistentes a meticilina y que dieron resultados negativos de RPC para mecA empleando los oligonucleótidos descritos por Murakami, y encontramos dos aislados portadores del homólogo descrito por García-Álvarez ${ }^{29}$. Este es el primer reporte de este tipo de homólogo de mecA en Paraguay y pone de manifiesto la variabilidad genética de las cepas de $S$. aureus circulantes en el país. Los nueve aislados restantes podrían portar otros homólogos de mecA o bien otros mecanismos que confieran resistencia a los antimicrobianos $\beta$-lactámicos, cuestión que estudiaremos próximamente.
La presencia del gen $l u k-P V$ se dio tanto en aislados SARM como en SASM. Este factor de virulencia ha sido ampliamente descrito en aislados resistentes a meticilina, pero su rol en infecciones causadas por SASM se ha estudiado de forma reciente en distintas latitudes ${ }^{31,37,38}$. Así, hay reportes de SASM portadores de $l u k-P V$, tanto a nivel internacional como regional. Rasigade y cols., analizaron la presencia de $l u k-P V$ en aislados de SASM que habían sido remitidos al Centro Nacional de Referencia de Staphylococcus en Francia durante 26 años (19812007), provenientes de 19 países, con fines de tipificar molecularmente las toxinas presentes en los mismos. Del total de 211 aislados SASM luk- $P V$ positivos, cuatro provenían de Paraguay y fueron remitidos al laboratorio francés en el período 2001 a $2005^{39}$. Así mismo un trabajo realizado en trabajadores de la salud del Hospital General Pediátrico Niños de Acosta Ñú mostró una frecuencia de $4,8 \%$ de $l u k-P V$, siendo $100 \%$ de estos aislados portadores de $l u k-P V$ sensibles a meticilina ${ }^{32}$.

Se pudo observar que aquellos pacientes con infecciones por cepas de SASM-PVL positivo presentaban una mayor tendencia a presentar infecciones invasoras que aquellas infecciones producidas por cepas de SASMPVL negativas $[\mathrm{p}=0,08, \mathrm{RR} 1,88$ (IC 0,9-3,8)]. En este sentido, en la actualidad existe controversia acerca del papel de PVL en la patogenicidad de la infección por $S$. aureus. Voyich y cols., argumentan que PVL no es necesario para la patogénesis de $S$. aureus en sepsis, bacteriemia o en abscesos de piel; así mismo, Yanhong y cols., estudiando aislados de $S$. aureus causantes de infecciones invasoras en niños chinos, sostienen que el gen de pvl no tiene un papel crucial en la patogénesis. Por otra parte, Labandeira-Rey y cols., realizaron un trabajo en modelo murino y demostraron que la presencia de la proteína PVL es suficiente para producir neumonía y que induce cambios de expresión en $S$. aureus aumentando los niveles del factor inflamatorio pulmonar (proteína Spa). Un estudio realizado en España analizando la sobrevida de pacientes con neumonía causada por $S$. aureus mostró que la presencia de PVL aumentaba 1,56 veces el riesgo de muerte, siendo estadísticamente significativa ${ }^{40-43}$.

En conclusión, este estudio constituye la primera caracterización fenotípica y genotípica de $S$. aureus, tanto resistentes como sensibles a meticilina, provenientes de infecciones adquiridas en la comunidad en población pediátrica y realizada en la comunidad de Paraguay. Si bien este trabajo presenta limitantes como el tamaño de muestra, es un punto de inicio para el diseño de próximos estudios de cohortes prospectivos para evaluar los factores de riesgo y el pronóstico de los pacientes con infecciones por SARM, así como aquellas infecciones producidas por SASM-PVL positivas.

En un futuro próximo se desea llegar a la identifica- 
ción de los clones correspondientes, tanto a los aislados, SARM como SASM, mediante el empleo de métodos moleculares como la tipificación del cassette SCCmec y la secuenciación correspondiente para el MLST. Así mismo como identificar el mecanismo que confiere resistencia a meticilina presente en aquellos aislados que presentan el fenotipo pero en los que el gen $m e c A$ no ha podido ser amplificado con los oligonucleótidos empleados en este trabajo. Los estudios epidemiológicos que nos permitan evidenciar las características genéticas de las cepas que circulan en nuestra región son de suma importancia no sólo para cubrir lagunas en el conocimiento de nuestra epidemiología sino para fortalecer los sistemas de vigilancia de bacterias multi-resistentes, que nos permitan la elección de las mejores medidas de manejo de nuestros pacientes.

Agradecimientos. A Laura Maldonado, Magdalena Rodríguez, Mirian Villagra y Gloria Peralta, Hospital General Pediátrico, por su colaboración para el llenado de las fichas clínicas de los aislados. A Gabriel Gutkind de la Facultad de Farmacia de la UBA, Argentina por ceder gentilmente aislados previamente identificados que sirvieron de controles positivos. A Norma Fariña del Dpto. de Análisis Clínicos y Microbiología por su ayuda en la evaluación de los aislados hetero-resistentes frente a oxacilina.

\section{Resumen}

Introducción: La prevalencia de infecciones por Staphylococcus aureus en la comunidad ha aumentado, siendo la población pediátrica la más afectada; poniendo de relieve la necesidad de una vigilancia epidemiológica. Objetivo: Caracterizar clínica, fenotípica y genotípicamente aislados de $S$. aureus de muestras de niños con infecciones adquiridas en la comunidad, recolectadas en hospitales de Asunción y el Departamento Central, entre noviembre de 2009 y diciembre de 2010. Materiales y Métodos: Estudio descriptivo de corte trasverso. Datos clínicos fueron recabados de fichas, la susceptibilidad a antimicrobianos se hizo según criterio del CLSI y la detección de genes mec $A$ y luk- $P V$ se realizó por RPC empleando oligonucleótidos específicos. Resultados: De 123 aislados de S. aureus, $76 \%$ provenían de infecciones de piel y tejidos blandos y $20 \%$ de pacientes con bacteriemias. $18,7 \%$ (n: 23 ) fueron resistentes a meticilina (SARM). Se detectó la presencia de genes $m e c A$, una variante del mismo y $l u k-P V$ en $9,8 \%$, 1,6 y $39 \%$ de los aislados, respectivamente. El $43 \%$ de los SARM (n: 10) fue portador de $l u k-P V$. Las diferencias clínicas y demográficas entre pacientes infectados por SARM o SASM no fueron estadísticamente significativas. Discusión: Este estudio constituye la primera caracterización clínica, fenotípica y genotípica de $S$. aureus asociados a la comunidad en población pediátrica realizada en Paraguay.

\section{Referencias bibliográficas}

1.- Bartlett A H, Hulten K G. Staphylococcus aureus pathogenesis: secretion systems, adhesins, and invasins. Pediatr Infect Dis J 2010; 29 (9): 860-1.

2.- Kilic A, Li H, Stratton C W, Tang Y-W. Antimicrobial susceptibility patterns and staphylococcal cassette chromosome mec types of, as well as Panton-Valentine leukocidin occurrence among, methicillin-resistant Staphylococcus aureus isolates from children and adults in middle Tennessee. J Clin Microbiol 2006; 44 (12): 4436-40.

3.- Leeuwen $\mathrm{W}$ van, Nieuwenhuizen $\mathrm{W}$ van, Gijzen C, Verbrugh H, Belkum A van. Population studies of methicillin-resistant and-sensitive Staphylococcus aureus strains reveal a lack of variability in the $a g r \mathrm{D}$ gene, encoding a staphylococcal autoinducer peptide. J Bacteriol 2000; 182 (20): 5721-9.

4.- Chan P F, Foster S J. The role of environmental factors in the regulation of virulencedeterminant expression in Staphylococcus aureus 8325-4. Microbiology 1998; 144 (9): 2469-79.
5.- Palombarani S, Gardella N, Tuduri A, Figueroa S, Sly G, Corazza R, et al. Community-acquired methicillin-resistant Staphylococcus aureus infections in a hospital for acute diseases. Rev Argent Microbiol 2007; 39 (3): 151-5.

6.- Brady J M, Stemper M E, Weigel A, Chyou P-H, Reed K D, Shukla S K. Sporadic «Transitional» community-associated methicillin-resistant Staphylococcus aureus strains from health care facilities in the United States. J Clin Microbiol 2007; 45 (8): 2654-61.

7.- Campbell K M, Vaughn A F, Russell K L, Smith B, Jiménez D L, Barrozo C P, et al. Risk factors for community-associated methicillinresistant Staphylococcus aureus infections in an outbreak of disease among military trainees in San Diego, California, in 2002. J Clin Microbiol 2004; 42 (9): 4050-3.

8.- Kaplan S L, Hulten K G, González B E, Hammerman W A, Lamberth L, Versalovic J, et al. Three-year surveillance of communityacquired Staphylococcus aureus infections in children. Clin Infect Dis 2005; 40 (12): 1785-91.

9.- Paganini M, Della L P, Muller O B, Ezcurra G,
Uranga M, Aguirre C, et al. Infecciones por Staphylococcus aureus resistente a meticilina adquiridas en la comunidad en niños antes sanos y en niños relacionados al hospital en la Argentina. Rev Chil Infectol 2009; 26 (5): 406-12.

10.- Kim E S, Song J S, Lee H J, Choe P G, Park K H, Cho J H, et al. A survey of community-associated methicillin-resistant Staphylococcus aureus in Korea. J Antimicrob Chemother 2007; 60 (5): 1108-14.

11.- Echevarría Zárate J, Iglesias Quilca D. Estafilococo meticilino resistente, un problema actual en la emergencia de resistencia entre los Gram positivos. Rev Medica Hered 2003; 14 (4): 195-203.

12.- Kilic A, Guclu A U, Senses Z, Bedir O, Aydogan H, Basustaoglu A C. Staphylococcal cassette chromosome mec (SCCmec) characterization and Panton-Valentine leukocidin gene occurrence for methicillinresistant Staphylococcus aureus in Turkey, from 2003 to 2006. Antonie Van Leeuwenhoek 2008; 94 (4): 607-14.

13.- Martínez-Aguilar G, Ávalos-Mishaan A, Hulten K, Hammerman W, Mason EOJ, 
Kaplan S L. Community-acquired, methicillinresistant and methicillin-susceptible Staphylococcus aureus musculoskeletal infections in children. Pediatr Infect Dis J 2004; 23 (8): 701-6.

14.- Ma XX, Galiana A, Pedreira W, Mowszowicz M, Christophersen I, Machiavello S, et al. Community-acquired methicillin-resistant Staphylococcus aureus, Uruguay. Emerg Infect Dis 2005; 11 (6): 973-6.

15.- Paganini H, Firpo V, Villa A, Debbag R, Berberian G, Casimir L, et al. Clinical study and analysis of risk factors for mortality in 86 cases of infectious endocarditis in children and adolescents in Argentina: 1988-2000. Enf Infec Microbiol Clín 2004; 22 (8): 455-61.

16.- Boyle-Vavra S, Daum R S. Communityacquired methicillin-resistant Staphylococcus aureus: the role of Panton-Valentine leukocidin. Lab Investig J Tech Methods Pathol 2007; 87 (1): 3-9.

17.- Ma XX, Ito T, Tiensasitorn C, Jamklang M, Chongtrakool P, Boyle-Vavra S, et al. Novel type of staphylococcal cassette chromosome mec identified in community-acquired methicillin-resistant Staphylococcus aureus strains. Antimicrob Agents Chemother 2002; 46 (4): $1147-52$

18.- Coombs G W, Nimmo G R, Bell J M, Huygens F, O’Brien F G, Malkowski M J, et al. Genetic diversity among community methicillin-resistant Staphylococcus aureus strains causing outpatient infections in Australia. J Clin Microbiol 2004; 42 (10): 4735-43.

19.- Mera R M, Suaya J A, Amrine-Madsen H, Hogea C S, Miller L A, Lu E P, et al. Increasing role of Staphylococcus aureus and communityacquired methicillin-resistant Staphylococcus aureus infections in the United States: A 10-year trend of replacement and expansion. Microb Drug Resist 2011; 17 (2): 321-8.

20.- Galiana Villar Á. Infección por Staphylococcus aureus meticilino resistente adquirido en la comunidad. Arch Pediatría Urug 2003; 74 (1): 26-9.

21.- Reyes J, Rincón S, Díaz L, Panesso D, Contreras G A, Zurita J, et al. Dissemination of methicillin-resistant Staphylococcus aureus USA300 sequence type 8 lineage in Latin America. Clin Infect Dis 2009; 49 (12): 1861-77.

22.- Organización Panamericana de la Salud. Informe Anual de la Red de Monitoreo/Vigilancia de la Resistencia a los Antibióticos, 2008. https://www. google.cl/webhp?sourceid=chromeinstant\&ion $=1 \&$ espv $=2 \&$ ie $=$ UTF- $8 \# \mathrm{q}=$ Organiz aci\%C3\%B3n+Panamericana+de+la+Salud. +In forme + Anual + de + la + Red + de + Monitoreo $\% 2 \mathrm{FV}$ igilancia + de + la + Resistencia $+a+l o s+$ Antibi $\%$ C3 $\%$ B3ticos $\% 2 \mathrm{C}+2008 .+2008$

23.- Samudio G, Román O, Carnibella M. Infecciones por Staphylococcus aureus en pacientes en edad pediátrica internados en el Hospital Nacional de Itaguá. Universidad Católica Nuestra Señora de la Asunción; 2009.

24.- Sanabria G, Apodaca S, Ayala E, Lovera D, Arbo A. Community-acquired skin and soft-tissue Staphylococcus aureus infections in children hospitalized in referral center in Paraguay: eight years of surveillance. http:// www.imt.edu.py/admin/uploads/Documento/ sau.pdf

25.- Departamento de Prensa Ministerio de Salud Pública y Bienestar Social. Alerta epidemiológica por Staphylococcus aureus resistente a vancomicina [Internet]. Ministerio de Salud Pública y Bienestar Social; 2013 feb. Disponible en: www.mspbs.gov.py/v2/17902Alerta-epidemiologica-por-Staphylococcusaureus-resistente-a-vancomicina

26.- Zhang K, Sparling J, Chow B L, Elsayed S, Hussain Z, Church D L, et al. New quadriplex PCR assay for detection of methicillin and mupirocin resistance and simultaneous discrimination of Staphylococcus aureus from coagulase-negative staphylococci. J Clin Microbiol 2004; 42 (11): 4947-55.

27.- Murakami K, Minamide W, Wada K, Nakamura E, Teraoka H, Watanabe $\mathrm{S}$ Identification of methicillin-resistant strains of staphylococci by polymerase chain reaction. J Clin Microbiol 1991; 29 (10): 2240-4.

28.- Lina G, Piémont $Y$, Godail-Gamot F, Bes M, Peter M-O, Gauduchon V, et al. Involvement of Panton-Valentine leukocidin-producing Staphylococcus aureus in primary skin infections and pneumonia. Clin Infect Dis 1999; 29 (5): 1128-32.

29.- García-Álvarez L, Holden M T G, Lindsay H, Webb C R, Brown D F J, Curran M D, et al. Methicillin-resistant Staphylococcus aureus with a novel mecA homologue in human and bovine populations in the UK and Denmark: a descriptive study. Lancet Infect Dis 2011; 11 (8): 595-603.

30.- Elegbe I A. Influence of seasonal and weather variation on the incidence of coagulase positive staphylococci isolates among Nigerians with boil infections. J R Soc Health 1983; 103 (3): 118-9.

31.- Sola C, Saka H A, Vindel A, Bocco J L. Emergence and dissemination of a communityassociated methicillin-resistant Panton-Valentine leukocidin-positive Staphylococcus aureus clone sharing the sequence type 5 lineage with the most prevalent nosocomial clone in the same region of Argentina. J Clin Microbiol 2008; 46 (5): 1826-31.

32.- Carpinelli L. Caracterización fenótípica y genotípica de Staphylococcus aureus aislados de trabajadores de la salud de un centro hospitalario de referencia nacional [Internet]. UNA. Dirección General de Postgrado. Dirección General de Investigación Científica y Tecnológica. IICS; 2011 [citado 30 de junio de 2014]. Disponible en: http://bases.bireme.br/ cgi-bin/wxislind.exe/iah/online/?IsisScript=iah/ iah.xis\&src=google\&base $=$ LILACS\&lang=p\&n extAction $=$ lnk\&exprSearch $=600322$ \&indexSear $\mathrm{ch}=\mathrm{ID}$

33.- Gardella N, Picasso R, Predari S C, Lasala M, Foccoli M, Benchetrit G, et al. Methicillinresistant Staphylococcus aureus strains in Buenos Aires teaching hospitals: replacement of the multidrug resistant South American clone by another susceptible to rifampin, minocycline and trimethoprim-sulfamethoxazole. Rev Argent Microbiol 2005; 37 (3): 156-60.

34.- Rodríguez-Noriega E, Seas C, GuzmánBlanco M, Mejía C, Álvarez C, Bavestrello $\mathrm{L}$, et al. Evolution of methicillin-resistant Staphylococcus aureus clones in Latin America. Int J Infect Dis 2010; 14 (7): e560-6.

35.- Stegger M, Wirth T, Andersen P S, Skov R L, De Grassi A, Simões P M, et al. Origin and evolution of European community-acquired methicillin-resistant Staphylococcus aureus. mBio 2014; 5 (5): e01044-14.

36.- Shore A C, Deasy E C, Slickers P, Brennan G, O’Connell B, Monecke S, et al. Detection of staphylococcal cassette chromosome mec type XI carrying highly divergent $m e c \mathrm{~A}, m e c \mathrm{I}, m e c \mathrm{R} 1$, blaZ, and $c c r$ genes in human clinical isolates of clonal complex 130 methicillin-resistant Staphylococcus aureus. Antimicrob Agents Chemother 2011; 55 (8): 3765-73.

37.- Muttaiyah S, Coombs G, Pandey S, Reed P, Ritchie S, Lennon D, et al. Incidence, risk factors, and outcomes of Panton-Valentine leukocidin-positive methicillin-susceptible Staphylococcus aureus infections in Auckland, New Zealand. J Clin Microbiol 2010; 48 (10): 3470-4.

38.- Sola C, Cortés P, Saka H A, Vindel A, Bocco J L. Evolution and molecular characterization of methicillin-resistant Staphylococcus aureus epidemic and sporadic clones in Cordoba, Argentina. J Clin Microbiol 2006; 44 (1): $192-$ 200.

39.- Rasigade J-P, Laurent F, Lina G, Meugnier H, Bes M, Vandenesch F, et al. Global distribution and evolution of Panton-Valentine leukocidin- 
positive methicillin-susceptible Staphylococcus aureus, 1981-2007. J Infect Dis 2010; 201 (10): 1589-97.

40.- Voyich J M, Otto M, Mathema B, Braughton K R, Whitney A R, Welty D, et al. Is PantonValentine leukocidin the major virulence determinant in community-associated methicillin-resistant Staphylococcus aureus disease? J Infect Dis 1 2006; 194 (12):
1761-70.

41.- Qiao Y, Ning X, Chen Q, Zhao R, Song W, Zheng Y, et al. Clinical and molecular characteristics of invasive community-acquired Staphylococcus aureus infections in Chinese children. BMC Infect Dis 2014; 14: 582.

42.- Labandeira-Rey M, Couzon F, Boisset S, Brown E L, Bes M, Benito Y, et al. Staphylococcus aureus Panton-Valentine leukocidin causes necrotizing pneumonia. Science 2007; 315 (5815): 1130-3.

43.- López-Aguilar C, Pérez-Roth E, MéndezÁlvarez S, Moreno A, Durán M C, Casanova $\mathrm{C}$, et al. Association between the presence of the Panton-Valentine leukocidin-encoding gene and a lower rate of survival among hospitalized pulmonary patients with staphylococcal disease. J Clin Microbiol 2007; 45 (1): 274-6. 